SCIREA Journal of Clinical Medicine

ISSN: 2706-8870

http://www.scirea.org/journal/CM

January 24, 2021

SCIREA

Volume 6, Issue 1, February 2021

\title{
Impact of age at appendectomy on development of HCC: A population-based cohort study
}

\section{Yang-Ming Lee ${ }^{1,2}$, Chew-Teng Kor ${ }^{2}$}

${ }^{1}$ Department of Medicine, Kaohsiung Medical University No. 100, Shih-Chuan 1st Road, Kaohsiung 807, Taiwan

${ }^{2}$ Internal Medicine Research Center, Changhua Christian Hospital, Changhua, Taiwan Author contributions: YM Lee designed this study and wrote the manuscript as well as provided final approval of the manuscript.; CT Kor analyzed the data

*Corresponding authors: Yang-Ming Lee, MD., PhD (125756@ech.org.tw) ; 135 Nanhsiao Street, Changhua, 500 Taiwan; Tel: +886-4-723-8595 ext.5889

Supportive foundations: This work was supported by Changhua Christian hospital

\begin{abstract}
Aim: The etiology and epidemiology of hepatocellular carcinoma (HCC) has been linked to the entero-ecosystem, involving microbiome and entero-hepatic endocrine system. The human appendix, a microbial reservoir for repopulating the gastrointestinal tract, has currently been considered a crucial part of the immune system. Thus, appendectomy may change gut microbial structure and immune
\end{abstract}


function, thereby increasing chronic liver diseases, including HCC. The object of this study is to investigate the association between appendectomy and the risk of HCC.

Methods: We analyzed a cohort of 12864 patients who underwent appendectomy between 1998 and 2013 based on the Taiwan National Health Insurance Program database. A comparison cohort of 51456 persons without appendectomy was selected randomly and matched by sex, age, comorbidities, and index year. To ensure reliability of the results, a sensitivity analysis using a propensity score-matched study was performed. We observed the subsequent development of HCC in both cohorts.

Results: Although the overall incidence of HCC in the appendectomy patients was $8.7 \%$ higher than that in the non-appendectomy patients, it was not statistically significant (95\% confidence interval [CI], 0.833-1.417) after the adjustment of confounding factors. Multivariable regression analysis revealed that the adjusted hazard ratio (HR) of HCC was 1.870 for the appendectomy patients at age $<40$ years (95\% CI 1.012, 3.454) compared to the non-appendectomy patients. The incidence of HCC was higher within 5 years of post-appendectomy follow-up than for the non-appendectomy patients (HR of 1.771, 95\% CI, 1.143-2.674). Age affected the association between appendectomy and HCC risk $\left(\mathrm{P}_{\text {interaction }}=0.0207\right)$; in contrast, sex did not influence the association between appendectomy and HCC risk $\left(\mathrm{P}_{\text {interaction }}=\right.$ $0.5726)$.

Conclusions: The results of our study suggest that appendectomy increases HCC risk, particularly when executed before middle age.

Keywords: Appendectomy, hepatocellular carcinoma (HCC), enteric microbiota

\section{Introduction:}

Liver cancer is a complex disease related to multiple factors, including viral hepatitis, alcohol, aflatoxin, sex, and diabetes...etc. Liver cancer arise mainly in those cases with liver cirrhosis through a multi-step process involving hepatocellular injury, inflammation and compensatory regeneration. However, the fundamental mechanisms 
underlying the disease remain unclear. Remarkably, emerging evidence demonstrated the gut microbiota play a role in hepatocarcinogenesis. The human intestine is colonized by an estimated $10^{14}$ microrbes, which constitute a dynamic and diverse ecosystem. The gut microbial composition could be changed by age, geography, diet, alcohol and medications (1-3). After million-years of coevolution, the gut microbiota establishes a symbiotic relationship with host and impacts on human health as well as disease via maintain intestinal stability and immunomodulation.

Owing to its close anatomical connection with the intestine by the portal vein, liver is the first responder to expose gut microbiota and their products. As microbial ecosystem homeostasis, their harmful exposure to liver is minimizing. However, intestinal barrier disruption and dysbiosis contribute to chronic hepatic inflammation, subsequently leading to the development of HCC (4). Animal studies have revealed the gut microbiota promote HCC development via lipopolysaccharide (LPS)-Toll-like receptor 4 (TLR4) signaling (5) and LPS accumulation protects carcinogen-induced apoptosis and promotes liver tumorigenesis in animal model of liver cancer (6). Additionally, the significant differences in gut microbiota distribution exist during the development of chronic liver diseases (7).

Despite long being recognized as an evolutionary inherent organ, the human appendix nowadays has considered a critical part of the immune system. Furthermore, the human appendix may behave as a "safe house" to sustain beneficial bacteria in the intestine via immune-mediated biofilm formation (8-11). Therefore, appendectomy may affect the gut microbial composition and immune function, thereby reinforcing the development of many inflammatory diseases, including colon cancer, Crohn's disease and rheumatoid arthritis etc. (12-17). Little is known about the association between appendectomy and HCC. The aim of this study is to investigate whether appendectomy increases the risk of HCC.

\section{Materials and methods:}

\section{Study populations}

We used a one-million-person random sample from people enrolled in the Taiwan 
National Health Insurance Program (NHIP). This program started in 1995, and includes $99 \%$ of the 23 million-plus people who live in Taiwan. This database from the NHIP is managed and established for investigators. With the hospitalization database from the Taiwan NIHP, the appendectomy group was determined as patients aged 20 to 84 years with undergoing appendectomy from 1998 to 2013 (the International Classification of Diseases [ICD] 9th Revision, ICD-9 procedure codes 47.0 and 47.1. We identified a study group of 15917 patients with appendectomy between 1998 and 2013. We excluded 69 persons with history of HCC before the index date, 1366 patients with history of any other cancer before the index date, 4 patients with biliary cirrhosis before the index date, 24 patients with alcoholic cirrhosis before the index date, 145 patients with alcoholic liver disease before the index and 1455 patients who did not survive or follow-up $<18$ months. The remaining 12864 patients were included in the study as appendectomy cohorts. The index year was determined as the year of undergoing appendectomy. We established a random frequency-matched study cohort for assessing the association between appendectomy and HCC. For each identified appendectomy patient, four control groups were frequency matched by age, sex, and index year. A total of 12864 appendectomy patients and 51456 controls were included in the study cohort (Fig. 1). We also performed a propensity score-matched sensitivity analysis to ensure the reliability of our results. Propensity scores were calculated using multivariate logistic regression to predict the probability of $\mathrm{HCC}$ occurrence to balance the covariate distribution in the appendectomy and control groups. Based on the propensity score, 1:4 propensity score matching was performed in the sensitivity analysis.

All comorbidities were diagnosed with ICD-9 codes. The comorbidities potentially related to HCC were included as follows hypertension (ICD-9 401-405), hyperlipidemia (ICD-9 272), diabetes (ICD-9 250), obesity (ICD-9278, 783.1 and V77.8), hepatitis B infection (ICD-9070.20, 070.21, 070.22, 070.23, 070.30, 070.31, 070.32, 070.33, V02.61), hepatitis C infection (ICD-9 070.41, 070.44, 070.51, 070.54, 070.7, V02.62), alcoholism (ICD-9 291,303, 305.0, 357.5), diabetic drugs, statin, chronic hepatitis B treatment and chronic hepatitis $\mathrm{C}$ treatment. The major outcome was a new diagnosis of HCC (ICD-9 code 155) based on at least three outpatient 
diagnoses or one hospital discharge diagnosis during the follow-up period. All study subjects were followed until they were diagnosed with HCC at the end of 2013.

\section{Statistical analysis}

Demographic and clinical characteristics in the appendectomy and non-appendectomy subjects were presented as proportions and mean \pm standard deviation (SD). The standardized differences were used with an absolute value for the standardized difference of $>0.1$ considered to indicate important imbalances between the two groups for categorical or continuous matching variables, respectively. The incidence of HCC was measured as the event number of HCC analyzed during the follow-up year, divided by the total follow-up person-years for each subject.

The Cox proportional hazards regression model was applied to measure the hazard ratio (HR) and 95\% confidence interval (CI) for risk of $\mathrm{HCC}$ in the appendectomy compared with the non-appendectomy subjects. Multivariate Cox's analysis with cause-specific hazard models to estimate adjusted hazard ratios (aHRs) adjusted for confounder for appendectomy and other comorbidities including hypertension, hyperlipidemia, diabetes mellitus, obesity, alcoholism, tobacco-use, liver cirrhosis, hepatitis $\mathrm{B}$ infection and hepatitis $\mathrm{C}$ infection and medications (statins, antidiabetic agent, chronic hepatitis $\mathrm{B}$ treatment, chronic hepatitis $\mathrm{C}$ treatment). Because occurrence of HCC was rare-events, Firth's Penalized Likelihood method was used for all cause-specific Cox's proportional hazard models for bias-adjusted estimates.

Univariate and multivariate survival analysis to analyze the incident and relative risk of HCC. Subgroup analyses was used to distinguish between HCC risks in patients with appendectomy and non-appendectomy. Then, we used propensity score-based analysis to assess the reliability of our results. Propensity scores were calculated using multivariate logistic regression to predict the probability of HCC occurrence. Cumulative incidence curves from cause-specific hazard method for the appendectomy and non-appendectomy groups were also evaluated. 


\section{Results:}

There were 15917 patients in the appendectomy group and 12864 patients remained after the exclusion 69 patients with a history of HCC before index date, 1366 patients having any cancer before index date, 4 patients having biliary cirrhosis before index date, 24 patients having a history of alcoholic cirrhosis before index date, 145 patients having a history of alcoholic liver disease and 1455 patients who did not survive or follow-up $<18$ months. There were 51456 persons who did not receive appendectomy in the control group.

Comparisons in demographic characteristics are shown in Table 1; The mean ages of the

appendectomy and control groups were similar (33.75 years), and each was predominantly composed of males (52.34\%). In cohort 1 (random frequency-matched analysis), appendectomy and control groups were matched by hypertension, hyperlipidemia, diabetes, obesity, Hepatitis B infection, Hepatitis C infection, alcoholism, Tabacco, Cirrhosis, administration of statin, chronic HBV treatment and chronic HCV treatment. There was a slight disparity in Charlson's comorbidity index and number of visiting clinics between two appendectomy and control groups in cohort 1 .

In order to assess the reliability of cohort 1, propensity score-matched sensitivity analysis (cohort 2) was conducted and it revealed that appendectomy and control groups had equivalent distribution of all demographic characteristics. Overall, $0.55 \%$ $(n=71)$ of patients with appendectomy developed HCC versus $0.48 \%(n=246)$ of persons without appendectomy in cohort 1. Similar results were shown in Cohort 2. Using this data mining approach, we were able to explore the correlation between appendectomy and the development of HCC. As in Table 2, we found the overall incidence of HCC in the appendectomy patients was $18.4 \%$ higher than that in the control group, although the difference was not statistically significant $(\mathrm{P}=0.2102)$. The incidence of $\mathrm{HCC}$ in the appendectomy patients at age $<40$ years was 2.147 -fold greater than that in the control group $(95 \%$ CI 1.176, 3.92). The multivariable regression analysis disclosed that the adjusted HR of HCC was 1.870 for the 
appendectomy patients at age $<40$ years $(95 \%$ CI 1.012, 3.454) compared to controls. Age affected the association between appendectomy and HCC ( $\left.\mathrm{P}_{\text {interaction }}=0.0207\right)$; whereas, sex did not $\left(\mathrm{P}_{\text {interaction }}=0.5726\right)$.

Here, we used propensity score-based analysis to confirm the hazard ratio of HCC among patients $<40$ years of age, as shown in Table 3 . The propensity score-based analysis showed that adjusted HR of HCC in the appendectomy patients $<40$ years was $1.992(95 \%$ CI 1.071, 3.572) compared to controls.

Moreover, the cumulative incidences are shown in Fig 2. The adjusted HR of HCC in all patients with appendectomy was 1.087 (95\% CI, 0.83-1.42), that in patients $>40$ years of age with appendectomy was 0.983 (95\% CI, 0.73-1.33), and that in patients $<40$ years of age with appendectomy was 1.87 (95\% CI, 1.01-3.45) compared to controls.

The incidence of HCC stratified by follow-up period post-appendectomy is shown in Table 4 . The incidence within 5 years post-appendectomy follow-up was higher in the appendectomy patients with an HR of $1.771(\mathrm{P}=0.0085)$. In contrast, the incidence after 5 years post appendectomy follow-up was lower in the appendectomy group with an $\mathrm{HR}$ of $0.964(\mathrm{P}=0.8327)$.

\section{Discussion}

In this substantial retrospective population-based cohort study, we discovered no statistically significant association between appendectomy and HCC in the overall population (aHR, 1.087; 95\% CI, 0.833-1.417). However, appendectomy increased the risk of HCC among patients $<40$ years (aHR, 1.870; 95\% CI, 1.012-3.454). In the random frequency-matched cohort, all confounding factors except number of visiting clinics and Charlson Comorbidity Index score number of visiting clinics were equally assigned in the appendectomy and control groups. To diminish selection bias, a multivariate regression analysis was conducted of a propensity score-matched cohort, which disclosed that the adjusted HR of type HCC in the appendectomy patients $<40$ years was 1.992 (95\% CI, 1.071-3.572) compared to control groups. As a result, we 
concluded that appendectomy prior to 40 years of age is an independent risk factor for the development of HCC.

Aside from significant results in the age subgroup analyses confirmed by the different statistical methods, our results were adjusted by many confounding factors, including hypertension, hyperlipidemia, diabetes, obesity, Hepatitis B infection, Hepatitis C infection, alcoholism, Tabacco, Cirrhosis, administration of statin, chronic HBV treatment and chronic HCV treatment. This suggests that age indeed enforced a great effect on the association between appendectomy and HCC. Nonetheless, we identified that sex did not influence the association between appendectomy and HCC (Pinteraction $=0.572$ ). The aHR of HCC incidence in the male appendectomy group was $1.068(\mathrm{P}=0.680)$, while that in the female appendectomy group was $1.221(\mathrm{P}=$ $0.434)$ compared to controls.

As we know, appendicitis is one of the most common acute abdominal diagnoses, mainly derived from bacterial infection within the organ (18) and appendectomy remains the gold standard of treatment. Early reports have demonstrated appendectomy is associated with various human diseases $(12-17,19)$; however, the mechanism of their associations is not fully understood. Many factors might potentially contribute to its pathophysiology. First, appendicitis is a kind of bacterial infection. Second, the appendix now is regarded a safe house to control the commensal intestinal flora and an important part of the immune system. Third, prophylactic antibiotics is given before appendectomy surgery. Therefore, bacterial infection, appendectomy and prophylactic antibiotics use may disturb microbial composition, finally increasing HCC development. Based on the National Health Insurance Research Database (NHIRD), we explore the association with HCC development and appendectomy in real-world environment in a substantial population sampling in Taiwan and can help figure out if appendectomy is an appropriate resolution of appendicitis due to its long-term health effects.

Increasing evidence shows that the gut microbiota contributes progression of chronic liver diseases and finally HCC development $(5,20,21)$. Our animal study reveals that early exposure to gut microbiome reduces HCC risks in mice (22). Furthermore, some 
study shows that the gut microbial ecology linked to the progression of chronic liver disease may increase LPS level (7). Another study showed that Butyrate-producing bacterial genera were decreased, while genera producing lipopolysaccharide were expanded in early HCC versus healthy controls (23). Interestingly, more specific changes in the composition of the gut microbiota in various human diseases have been also identified (24). More studies need to be explored if any particular microbiome involve in the connection between appendectomy and HCC development.

Most significantly, our study results disclosed that appendectomy increases the risk of HCC, especially when completed in patients prior to middle age. Although gut dysbiosis may stimulate chronic inflammation, immune response may decline with aging. This might explain why the impact of age on appendectomy to HCC development. On the other hand, multiple risk factors may weaken the effect of appendectomy on the development of HCC later. Interestingly, this result is similar to our previous study, appendectomy increases type 2 diabetes risk before the middle age (19). This is compatible with increasing HCC derived from non-alcoholic fatty liver disease (NAFLD) (25), one of the main features of metabolic syndrome. Different time-to-exposure to gut microbiota may affecting HCC risks demonstrated in Germ free mice (22) may partially explain the effect on appendectomy to HCC development.

Regarding the prominent incidence within 5 years post-appendectomy follow-up, abrupt disruptions of the gut microbial structure might happen particularly at the early stage post-appendectomy owing to bacterial infection, antibiotics use and surgery. However, this effect due to appendectomy lasting only five years cannot explain more HCC development should occur after longer gut microbiota changes. Therefore, we wonder if these patients with appendicitis and appendectomy could originally have various composition of gut microbiota from normal population. Thus, they are inclined to have appendicitis and even have higher incidence of HCC in later life. On the other hand, we can infer if appendectomy was performed at earlier life and have more profound effect on the incidence of HCC in later life. But our results do not support our idea and cutoff age occurs at 40 years. Many contradictory points need to be clarified in the future 


\section{Study limitations}

There are some limitations in our study. First, stature, lifestyle data regarding dietary habits, exercise, socioeconomic status, and hereditary background were not available in this retrospective cohort study. Second, all comorbidities were diagnosed using ICD-9 codes, the certainty of which should be supported by more objective evidence, such as laboratory, image and pathological reports. Third, we have to consider perforated appendicitis different from non-perforated appendicitis, laparoscopic appendectomy distinct from open appendectomy and with or without any bacteremia complication etc.. Finally, except careful study design and adjustment for confounding factors, some selection bias may have come from the retrospective nature of this study.

\section{Conclusions}

In conclusion, appendectomy performed in patients $<40$ years is associated with the risk of HCC independent of hypertension, hyperlipidemia, diabetes, obesity, Hepatitis B infection, Hepatitis C infection, alcoholism, Tabacco, Cirrhosis, administration of statin, chronic HBV treatment and chronic HCV treatment. Further studies should be performed to clarify the role of appendectomy in the development of HCC.

\section{Acknowledgements}

We thank the Internal Medicine Research Center of Changhua Christian Hospital for supporting our study

\section{References}

[1] Haque TR, Barritt ASt. Intestinal microbiota in liver disease. Best Pract Res Clin Gastroenterol. 2016;30(1):133-42. 
[2] Backhed F, Ley RE, Sonnenburg JL, Peterson DA, Gordon JI. Host-bacterial mutualism in the human intestine. Science. 2005;307(5717):1915-20.

[3] Giannelli V, Di Gregorio V, Iebba V, Giusto M, Schippa S, Merli M, et al. Microbiota and the gut-liver axis: bacterial translocation, inflammation and infection in cirrhosis. World J Gastroenterol. 2014;20(45):16795-810.

[4] Schnabl B, Brenner DA. Interactions between the intestinal microbiome and liver diseases. Gastroenterology. 2014;146(6):1513-24.

[5] Dapito DH, Mencin A, Gwak GY, Pradere JP, Jang MK, Mederacke I, et al. Promotion of hepatocellular carcinoma by the intestinal microbiota and TLR4. Cancer Cell. 2012;21(4):504-16.

[6] Yu LX, Yan HX, Liu Q, Yang W, Wu HP, Dong W, et al. Endotoxin accumulation prevents carcinogen-induced apoptosis and promotes liver tumorigenesis in rodents. Hepatology. 2010;52(4):1322-33.

[7] Xie G, Wang X, Liu P, Wei R, Chen W, Rajani C, et al. Distinctly altered gut microbiota in the progression of liver disease. Oncotarget. 2016;7(15):19355-66.

[8] Randal Bollinger R, Barbas AS, Bush EL, Lin SS, Parker W. Biofilms in the large bowel suggest an apparent function of the human vermiform appendix. Journal of theoretical biology. 2007;249(4):826-31.

[9] Berry RJ. The True Caecal Apex, or the Vermiform Appendix: Its Minute and Comparative Anatomy. Journal of anatomy and physiology. 1900;35(Pt 1):83-100.9.

[10] Spencer J, Finn T, Isaacson PG. Gut associated lymphoid tissue: a morphological and immunocytochemical study of the human appendix. Gut. 1985;26(7):672-9.

[11] Laurin M, Everett ML, Parker W. The cecal appendix: one more immune component with a function disturbed by post-industrial culture. Anatomical record (Hoboken, NJ : 2007). 2011;294(4):567-79. 
[12] Wu SC, Chen WT, Muo CH, Ke TW, Fang CW, Sung FC. Association between appendectomy and subsequent colorectal cancer development: an Asian population study. PLoS One. 2015;10(2):e0118411.

[13] Andersson RE, Olaison G, Tysk C, Ekbom A. Appendectomy is followed by increased risk of Crohn's disease. Gastroenterology. 2003;124(1):40-6.

[14] Liao KF, Lai SW, Lin CL, Chien SH. Appendectomy correlates with increased risk of pyogenic liver abscess: A population-based cohort study in Taiwan. Medicine (Baltimore). 2016;95(26):e4015.

[15] Tzeng YM, Kao LT, Kao S, Lin HC, Tsai MC, Lee CZ. An appendectomy increases the risk of rheumatoid arthritis: a five-year follow-up study. PLoS One. 2015;10(5):e0126816.

[16] Lai SW, Lin CL, Liao KF, Tsai SM. Increased risk of pulmonary tuberculosis among patients with appendectomy in Taiwan. Eur J Clin Microbiol Infect Dis. 2014;33(9):1573-7.

[17] Sanders NL, Bollinger RR, Lee R, Thomas S, Parker W. Appendectomy and Clostridium difficile colitis: relationships revealed by clinical observations and immunology. World J Gastroenterol. 2013;19(34):5607-14.

[18] Swidsinski A, Dorffel Y, Loening-Baucke V, Theissig F, Ruckert JC, Ismail M, et al. Acute appendicitis is characterised by local invasion with Fusobacterium nucleatum/necrophorum. Gut. 2011;60(1):34-40.

[19] Lee YM, Kor CT, Zhou D, Lai HC, Chang CC, Ma WL. Impact of age at appendectomy on development of type 2 diabetes: A population-based cohort study. PLoS One. 2018;13(10):e0205502.

[20] Yoshimoto S, Loo TM, Atarashi K, Kanda H, Sato S, Oyadomari S, et al. Obesity-induced gut microbial metabolite promotes liver cancer through senescence secretome. Nature. 2013;499(7456):97-101.

[21] Ma C, Han M, Heinrich B, Fu Q, Zhang Q, Sandhu M, et al. Gut microbiome-mediated bile acid metabolism regulates liver cancer via NKT cells. Science. 2018;360(6391). 
[22] Lee Y-M, Chang W-C, Lei F-J, Kor C-T, Lai H-C, Chen Y-L, et al. Early Exposure to Gut Microbiome Reduces Hepatocellular Carcinoma Risks in Mice. BioMed Research International. 2020;2020:9807379.

[23] Ren Z, Li A, Jiang J, Zhou L, Yu Z, Lu H, et al. Gut microbiome analysis as a tool towards targeted non-invasive biomarkers for early hepatocellular carcinoma. Gut. 2019;68(6):1014-23.

[24] Ivanov, II, Honda K. Intestinal commensal microbes as immune modulators. Cell Host Microbe. 2012;12(4):496-508.

[25] Margini C, Dufour JF. The story of HCC in NAFLD: from epidemiology, across pathogenesis, to prevention and treatment. Liver Int. 2016;36(3):317-24. 